\title{
Structure of Self-Assembled Multilayers \\ Prepared from Water-Soluble Polythiophenes
}

\author{
Antti Viinikanoja, ${ }^{, 1,2}$ Sami Areva, ${ }^{2,3}$ Natalia Kocharova, ${ }^{1}$ Timo Ä̈̈ritalo, ${ }^{1}$ Maarit \\ Vuorinen, ${ }^{1}$ Arto Savunen, ${ }^{1}$ Jouko Kankare, ${ }^{1}$ and Jukka Lukkari ${ }^{*}$, \\ ${ }^{1}$ Department of Chemistry, University of Turku, FIN-20014 Turku \\ ${ }^{2}$ Graduate School of Materials Research (GSMR), Turku, Finland \\ ${ }^{3}$ Department of Physical Chemistry, Åbo Akademi University, FIN-20050 Åbo
}

\section{SUPPORTING INFORMATION}

\section{Contents}

${ }^{1} \mathrm{H}$ NMR and ${ }^{13} \mathrm{C}$ NMR data of thiophene monomers

NMR spectra

$$
\begin{aligned}
& { }^{1} \mathrm{H} \text { NMR } \\
& { }^{13} \mathrm{C} \text { NMR } \\
& \text { 3TOHIM } \\
& { }^{1} \mathrm{H} \text { NMR } \\
& { }^{13} \mathrm{C} \mathrm{NMR} \\
& \text { 4Me-3TOEIM } \\
& { }^{1} \mathrm{H} \text { NMR } \\
& \text { 4Me-3TOHIM } \\
& { }^{1} \mathrm{H} \text { NMR }
\end{aligned}
$$

$\mathrm{S} 2-\mathrm{S} 5$

S6-S8

S9-S12

S13-S15

S16-S19

S20-S23

Bulk IR-spectra of PTs and surface-IR spectra of (PT/PSS $)_{10}$ films

Bulk IR-spectra

P3TOPIM

P3TOHIM

S25

P4Me-3TOEIM

S26

P4Me-3TOHIM

S27

Surface IR-spectra in $v(\mathrm{CH})$ region

$(\mathrm{PT} / \mathrm{PSS})_{10}+$ peak deconvolution $\quad$ S28

$\begin{array}{ll}\text { Tentative assignment of IR-bands } & \text { S29 }\end{array}$

\section{AFM-results}

Density of PT-based particles in (PT/PSS $)_{10}$ films

S30 


\section{Viinikanoja et al : Supplemetary Information S1}

${ }^{1} \mathrm{H}$ NMR and ${ }^{13} \mathrm{C}$ NMR shifts $(\delta)$ in $\mathrm{DMSO} / \mathrm{D}_{2} \mathrm{O}$ and their assignments for the alkoxythiophene monomers (3TOPIM, 3TOHIM, 4Me-3TOEIM, 4Me-3TOHIM).

\section{TOPIM:}

${ }^{1} \mathrm{H}$ NMR (DMSO/D $\left.\mathrm{O}\right): 8.60(1 \mathrm{H}, \mathrm{s}), 7.40(1 \mathrm{H}, \mathrm{s}), 7.33(1 \mathrm{H}, \mathrm{s}), 7.27(1 \mathrm{H}, \mathrm{dd}), 6.67(1 \mathrm{H}$, dd), $6.42(1 \mathrm{H}, \mathrm{t}), 4.31(2 \mathrm{H}, \mathrm{t}), 3.99(2 \mathrm{H}, \mathrm{t}), 3.76(3 \mathrm{H}, \mathrm{s}), 2.25(2 \mathrm{H}, \mathrm{s})$

${ }^{13}$ C NMR: 156, 136, 126, 124, 122, 119, 99, 67, 47, 36, 29

See S2-S5 and S6-S8 for ${ }^{1} \mathrm{H}$ NMR and ${ }^{13} \mathrm{C}$ NMR spectra, respectively.

\section{TOHIM:}

${ }^{1} \mathrm{H}$ NMR(D $\left(\mathrm{D}_{2} \mathrm{O} / \mathrm{DMSO}\right): 8.57(1 \mathrm{H}, \mathrm{s}), 7.38(1 \mathrm{H}, \mathrm{s}), 7.33(1 \mathrm{H}, \mathrm{m}), 7.27(1 \mathrm{H}, \mathrm{dd}), 6.71(1 \mathrm{H}$, d), 6.44(1H, d), $4.09(2 \mathrm{H}, \mathrm{t}), 3.94(2 \mathrm{H}, \mathrm{t}), 3.77(3 \mathrm{H}, \mathrm{d}) 1.79(2 \mathrm{H}, \mathrm{m}), 1.66(2 \mathrm{H}, \mathrm{m}), 1.38$ $(2 \mathrm{H}, \mathrm{m}), 1.25(2 \mathrm{H}, \mathrm{m})$

${ }^{13} \mathrm{C}$ NMR $\left(\mathrm{D}_{2} \mathrm{O}\right): 157,138,126,124,122,119,99,71,49,38,29,28,25,24$

See S9-S12 and S13-S15 for ${ }^{1} \mathrm{H}$ NMR and ${ }^{13}$ C NMR spectra, respectively.

\section{Me-3TOEIM:}

${ }^{1} \mathrm{H}$ NMR (DMSO/D $\left.{ }_{2} \mathrm{O}\right): 9.07(1 \mathrm{H}, \mathrm{s}), 7.71(1 \mathrm{H}, \mathrm{t}), 7.64(1 \mathrm{H}, \mathrm{t}), 7.02(1 \mathrm{H}, \mathrm{dd}), 6.52(1 \mathrm{H}$, d), $4.56(2 \mathrm{H}, \mathrm{t}), 4.24(2 \mathrm{H}, \mathrm{t}), 3.83(3 \mathrm{H}, \mathrm{s}), 1.94(3 \mathrm{H}, \mathrm{s})$

See S16-S19 for ${ }^{1} \mathrm{H}$ NMR spectra.

\section{Me-3TOHIM:}

${ }^{1} \mathrm{H}$ NMR (DMSO/D $\left.\mathrm{D}_{2} \mathrm{O}\right): 8.60(1 \mathrm{H}, \mathrm{s}), 7.33(2 \mathrm{H}, \mathrm{d}), 6.81(1 \mathrm{H}, \mathrm{d}), 6.27(1 \mathrm{H}, \mathrm{d}), 4.06(2 \mathrm{H}$, t), $3.86(2 \mathrm{H}, \mathrm{d}), 3.77(3 \mathrm{H}, \mathrm{s}), 1.92(3 \mathrm{H}, \mathrm{s}), 1.75(2 \mathrm{H}, \mathrm{m}), 1.64(2 \mathrm{H}, \mathrm{m}), 1.35(2 \mathrm{H}, \mathrm{m}), 1.23$ $(2 \mathrm{H}, \mathrm{m})$

See S20-S23 for ${ }^{1} \mathrm{H}$ NMR spectra. 


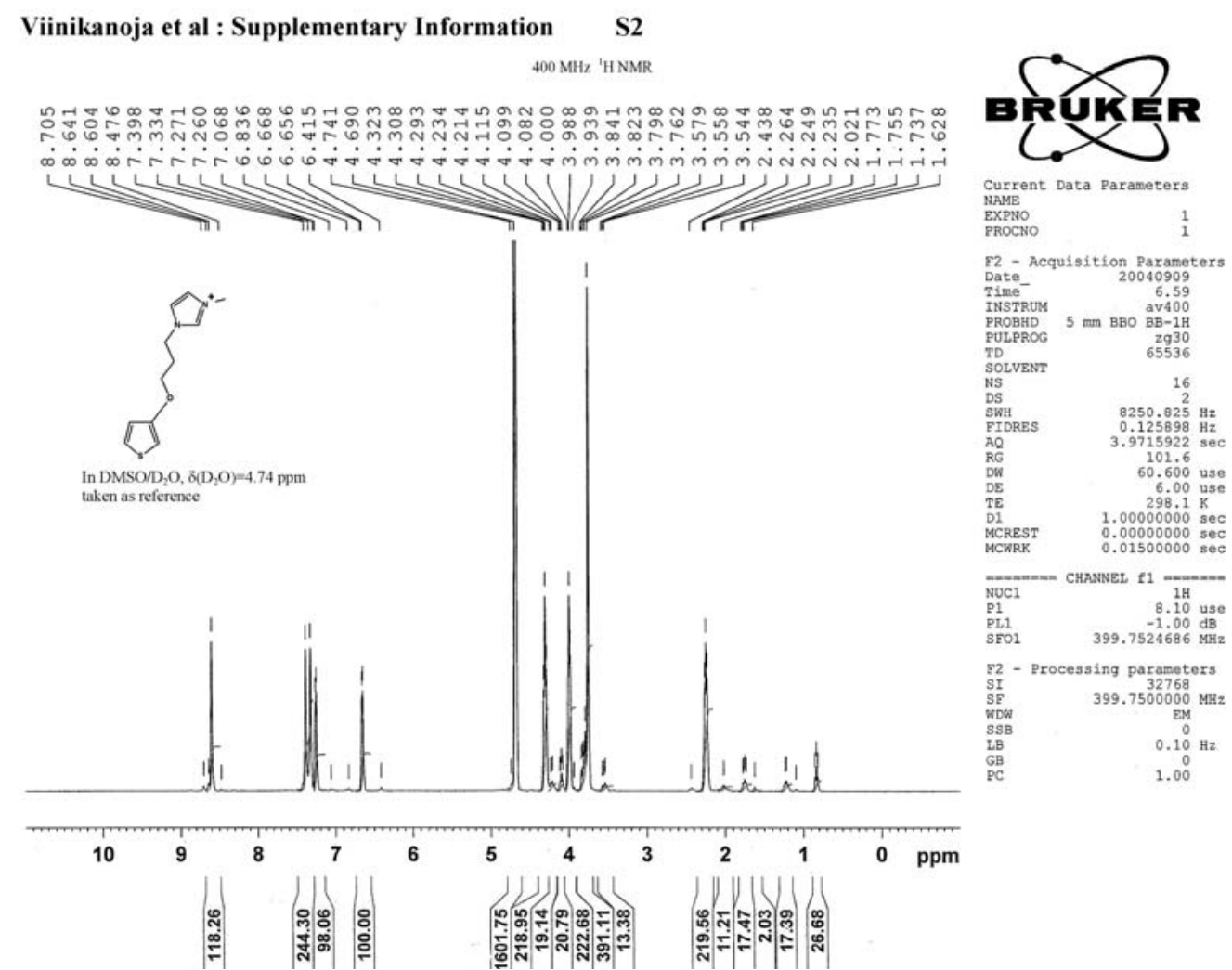

Viinikanoja et al : Supplementary Information S3
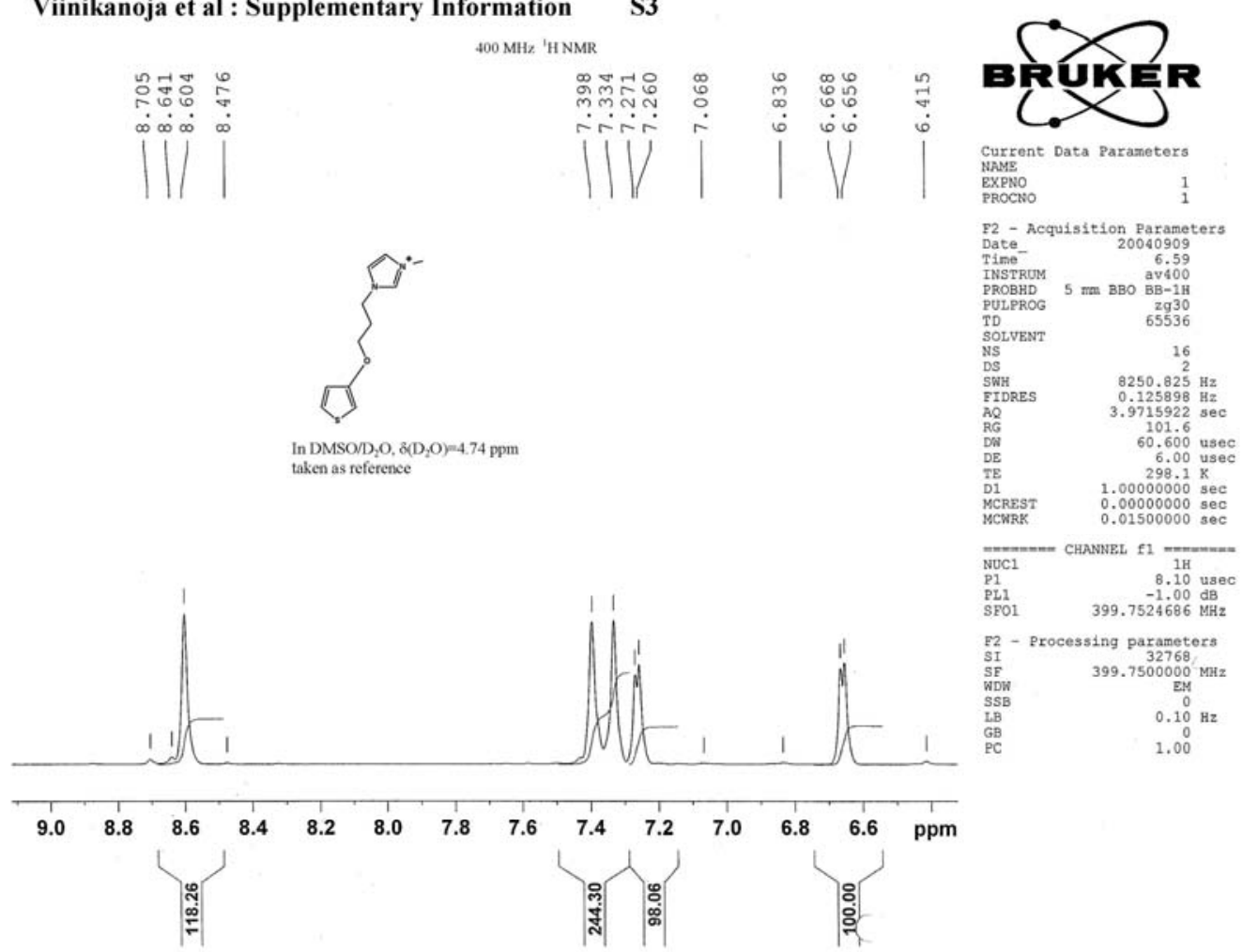
Viinikanoja et al : Supplementary Information S4

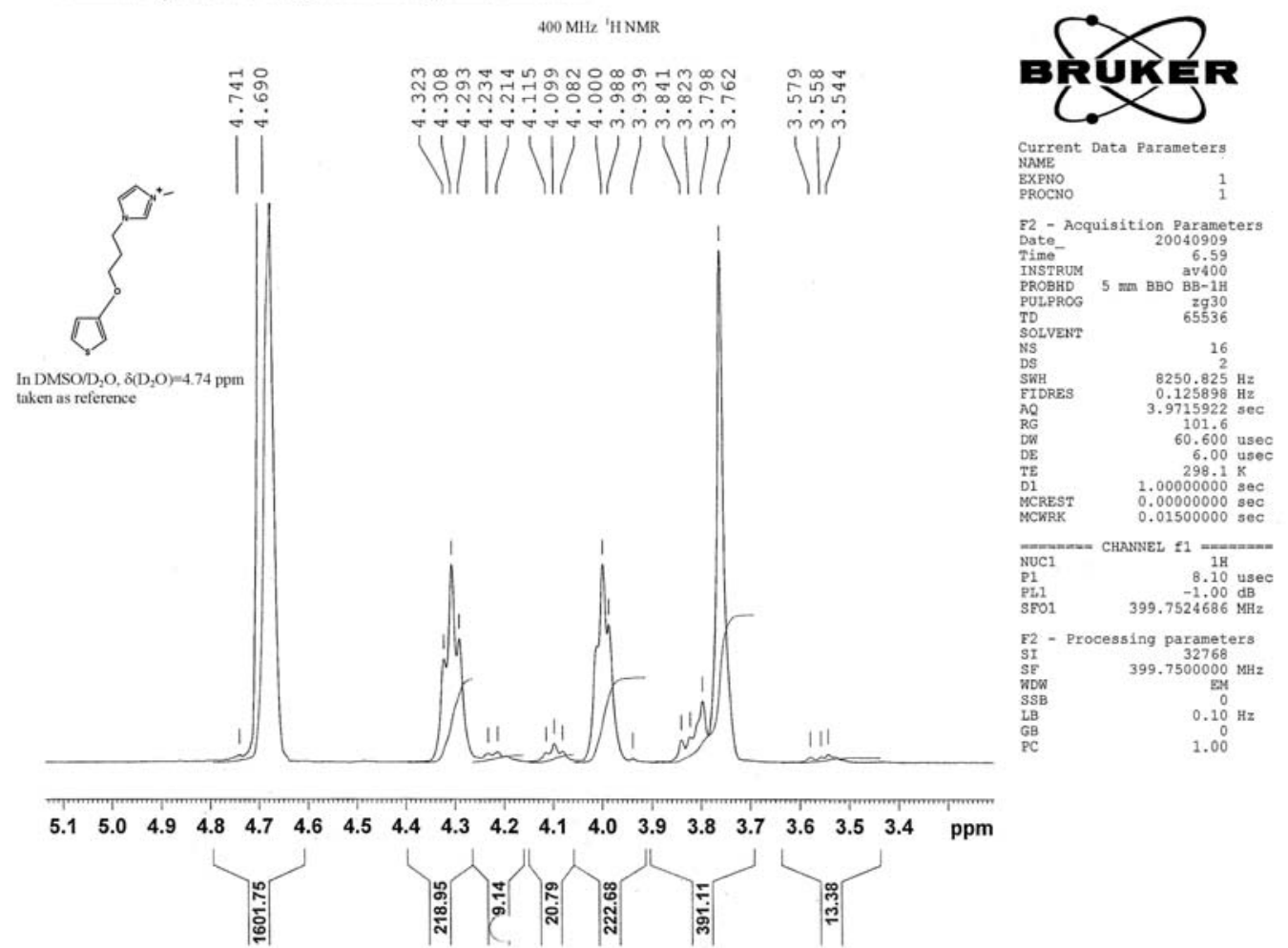

Viinikanoja et al : Supplementary Information S5

$400 \mathrm{MHz}$ 'H NMR
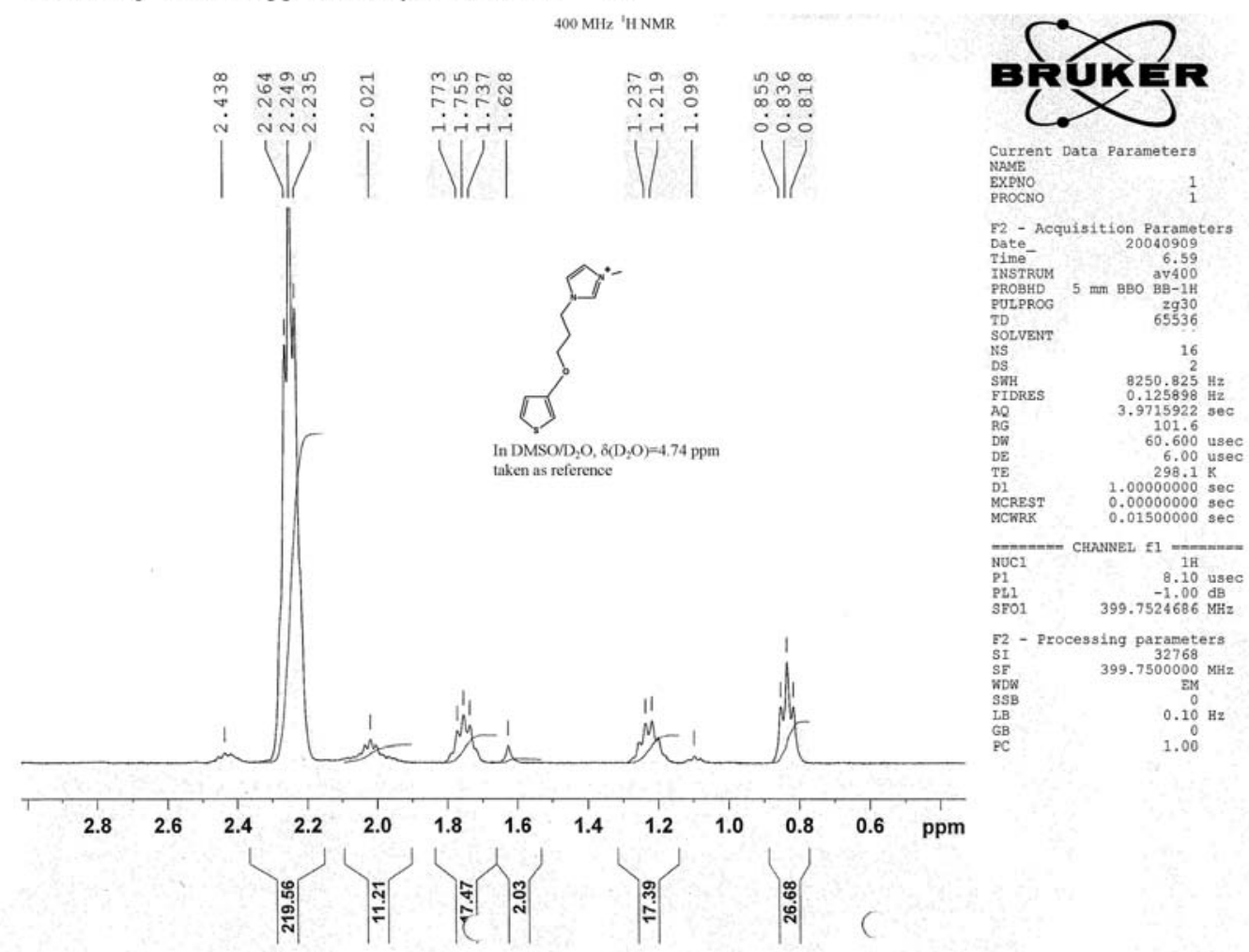


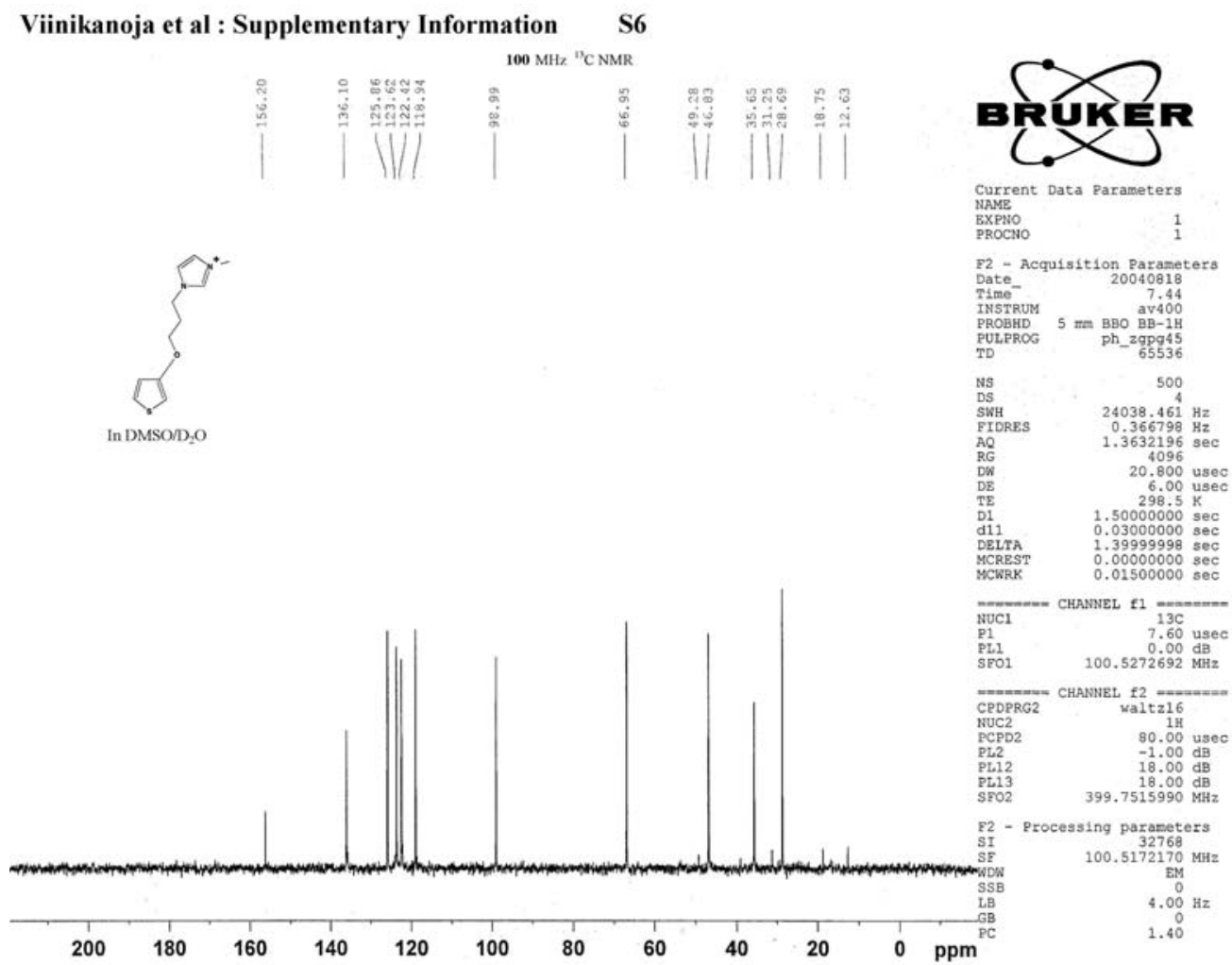

Viinikanoja et al : Supplementary Information $\quad$ S7

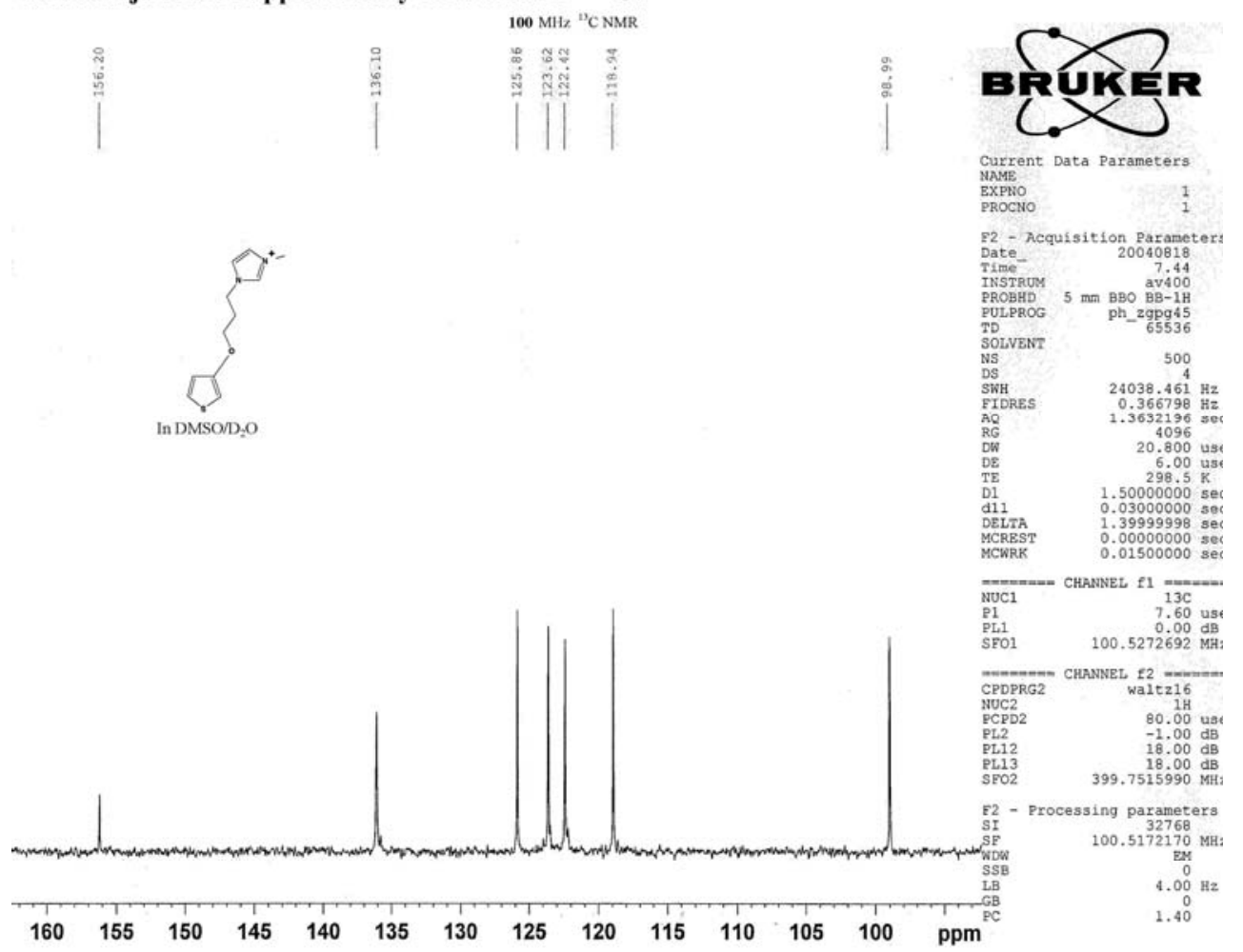




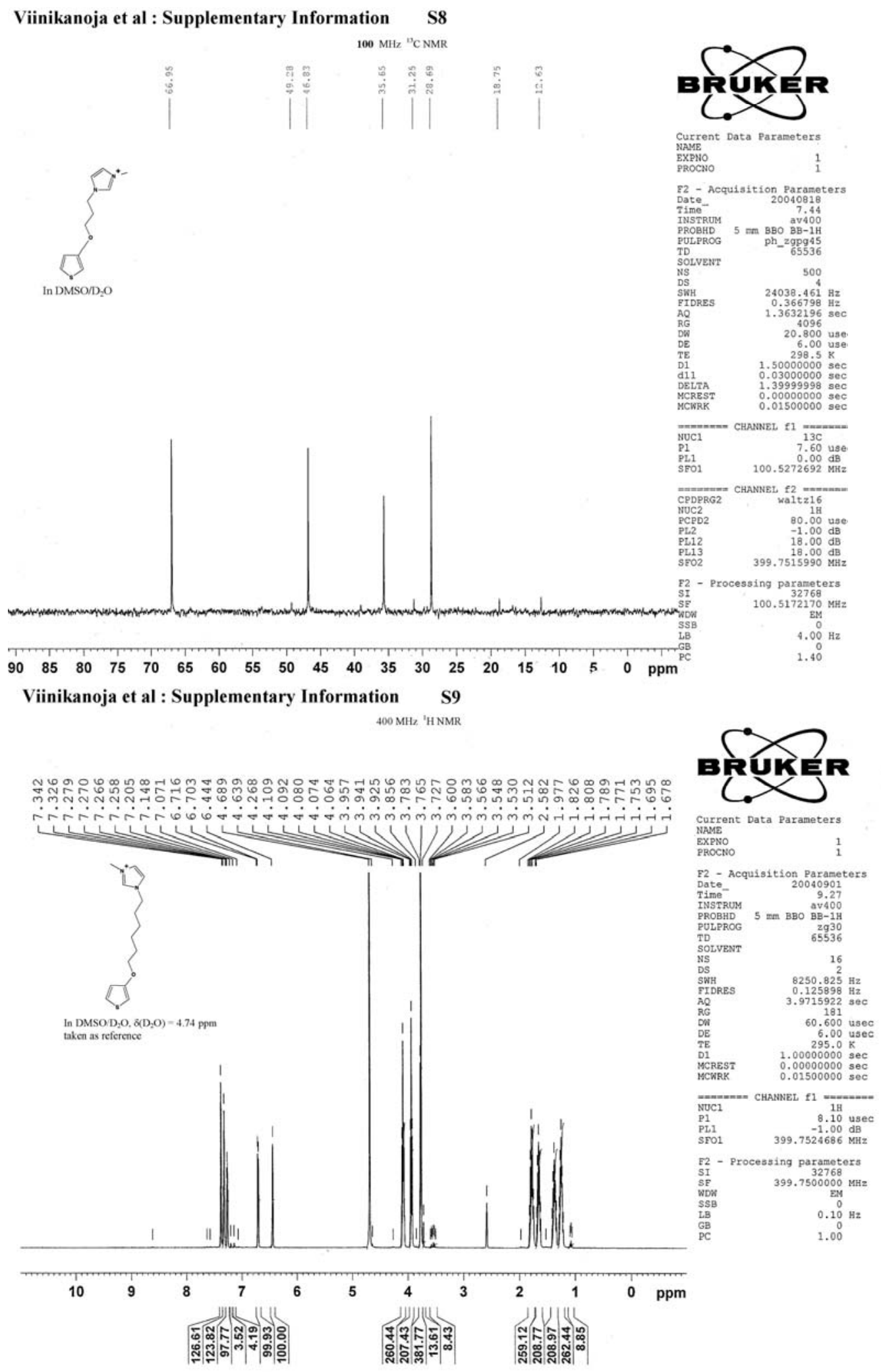



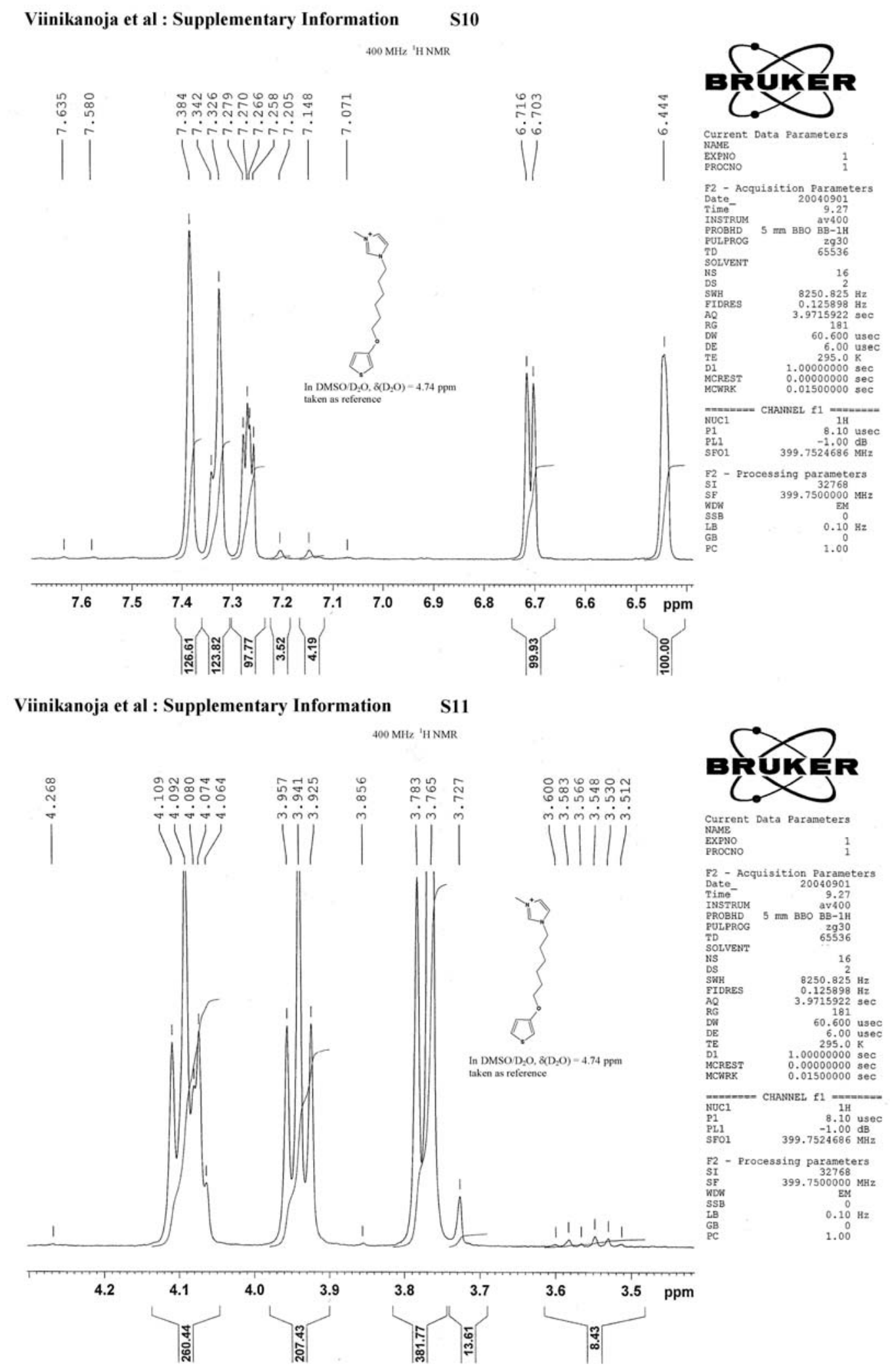


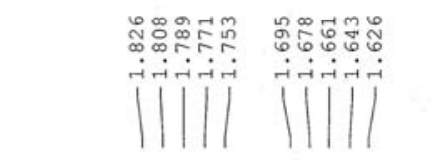

$$
\text { in }
$$

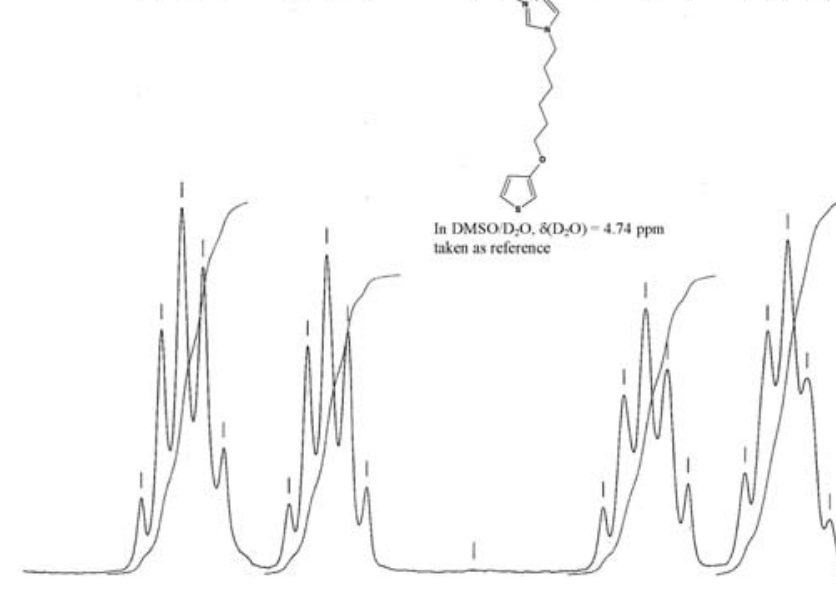$$
1.9
$$

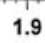

1.5
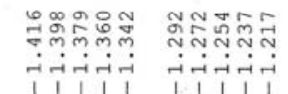
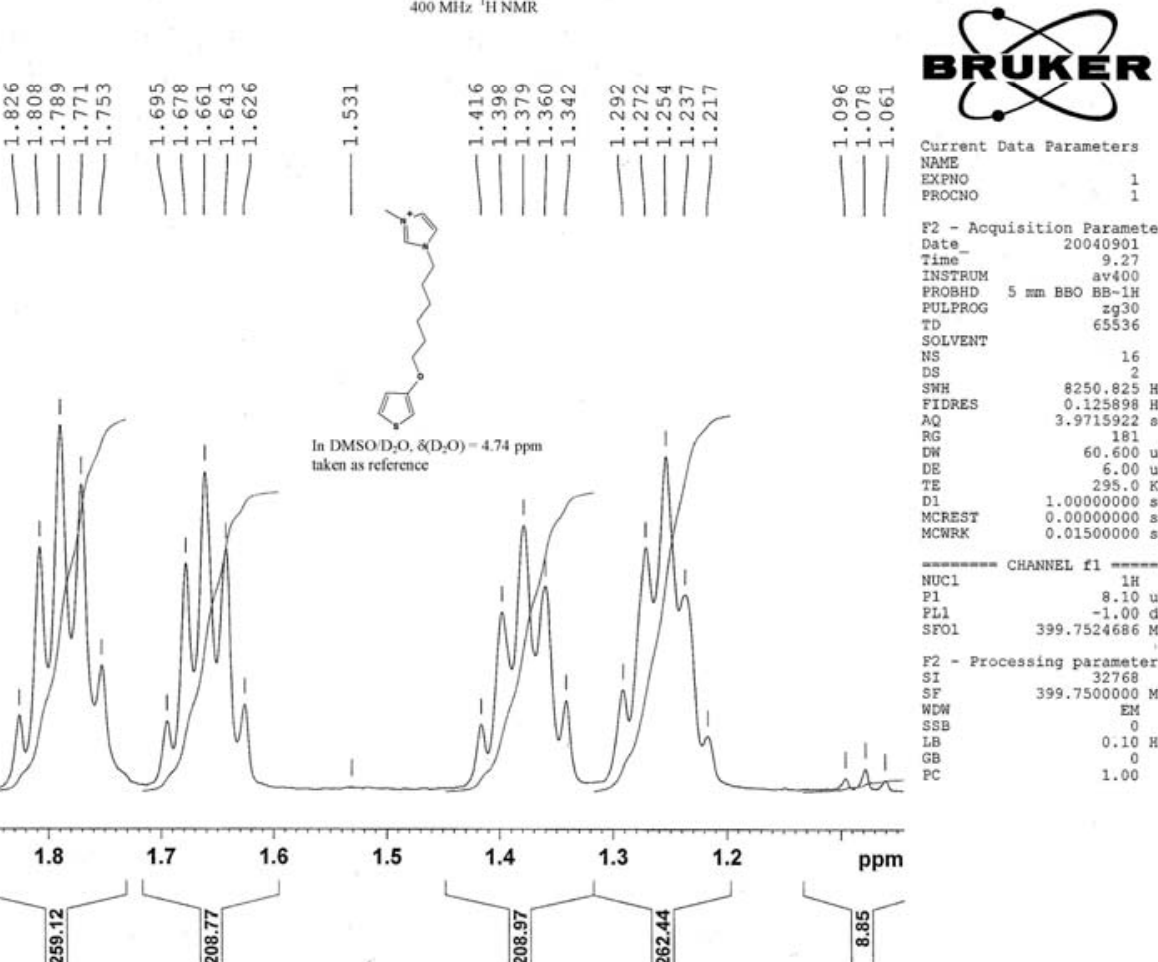

Current Data parameters
NaNE

ERPYO

F2 -
Dacquisition Parameters
7. 20040901

$\begin{array}{lr}\text { Date- } & 20040901 \\ \text { 7ime } & 9.27 \\ \text { INSTRUM } & \text { avio0 } \\ \text { PROBHD } & 5 \mathrm{~mm} \text { BBO BB-1H } \\ \text { PULPROG } & 2930 \\ \text { TD } & 65536\end{array}$

$\begin{array}{lr}\text { TD } & 65536 \\ \text { SOLVENT } & 16 \\ \text { WS } & 26 \\ \text { DS } & 8250.825 \\ \text { SWH } & \end{array}$

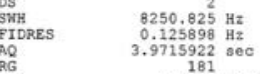

$\begin{array}{lr}\mathrm{D} & 60.600 \mathrm{usec} \\ \mathrm{DE} & 6.0 \mathrm{usec} \\ \mathrm{DE} & 29.0 \mathrm{~K} \\ \mathrm{TE} & 2.00 .00 \mathrm{sec}\end{array}$ $\begin{array}{ll}\text { D1 } & 1.00000000 \mathrm{sec} \\ \text { MCREST } & 0.00000000 \mathrm{sec} \\ \text { MCWRK } & 0.01500000 \mathrm{sec}\end{array}$

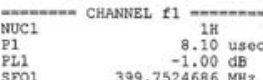

$7524686 \mathrm{MHz}$

$\begin{array}{lc}\text { F2 - Processing parameters } \\ \text { SI } \\ \text { SE } & 399.7500000 \mathrm{MHz} \\ \text { SD } & \text { EM } \\ \text { SSB } & 0 \\ \mathrm{SBB} & 0.10 \mathrm{~Hz} \\ \mathrm{LB} & 0 \mathrm{Bz} \\ \mathrm{GB} & \end{array}$

I' 1 a

1.00

Viinikanoja et al : Supplementary Information S13

$100 \mathrm{MHz}{ }^{13} \mathrm{CNMR}$

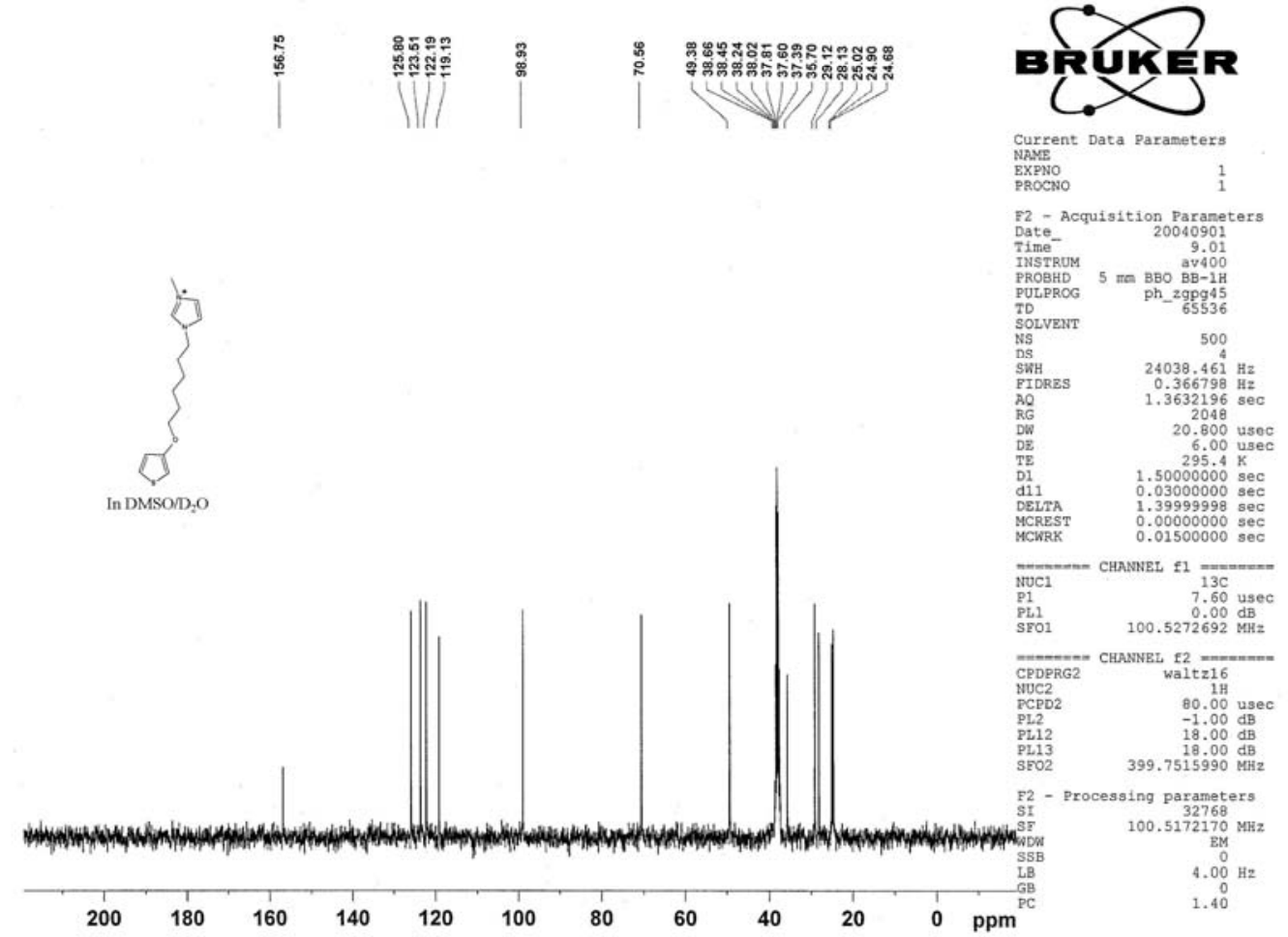


$100 \mathrm{MHz}{ }^{10} \mathrm{C} \mathrm{NMR}$

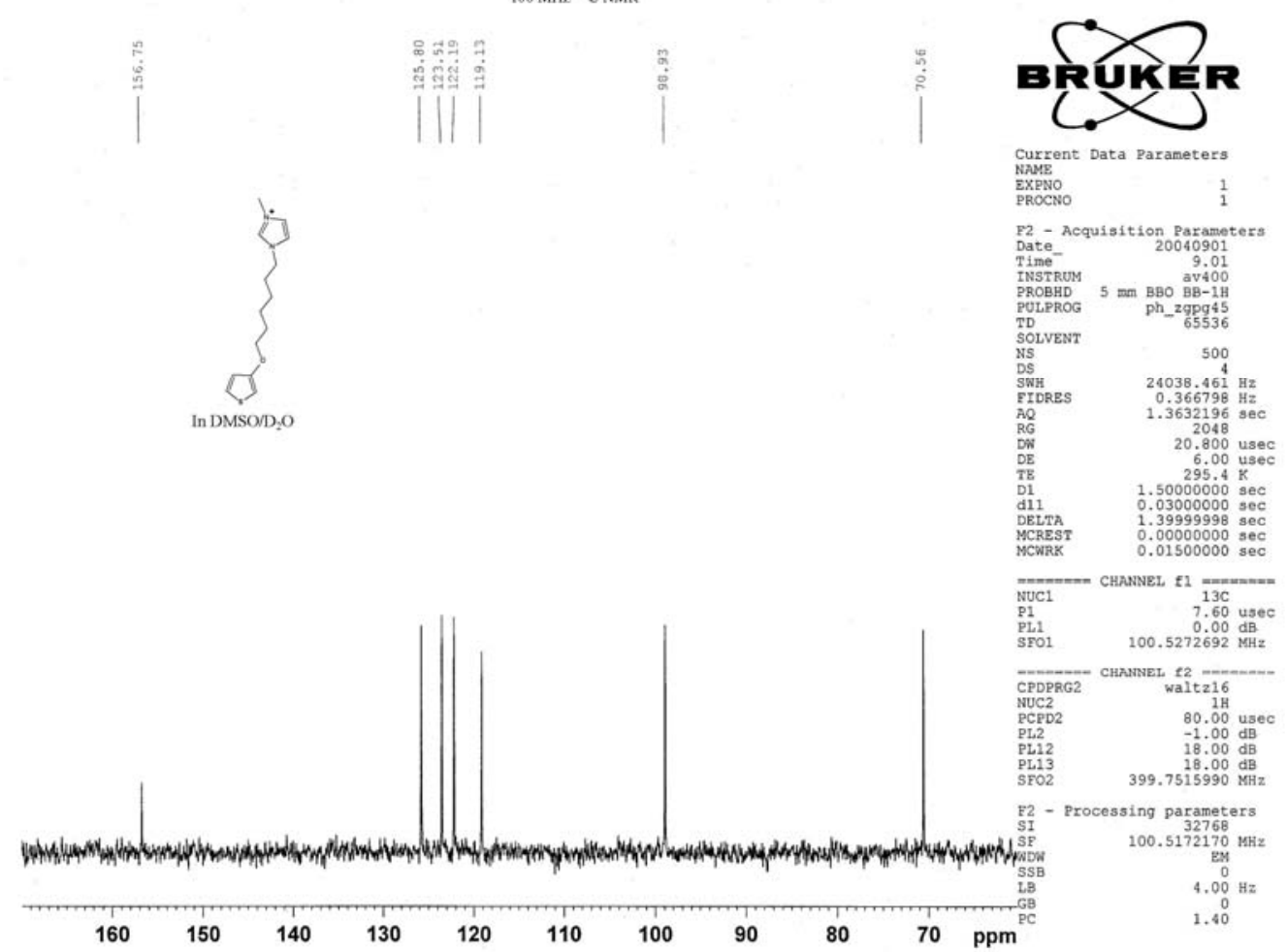

Viinikanoja et al : Supplementary Information S15

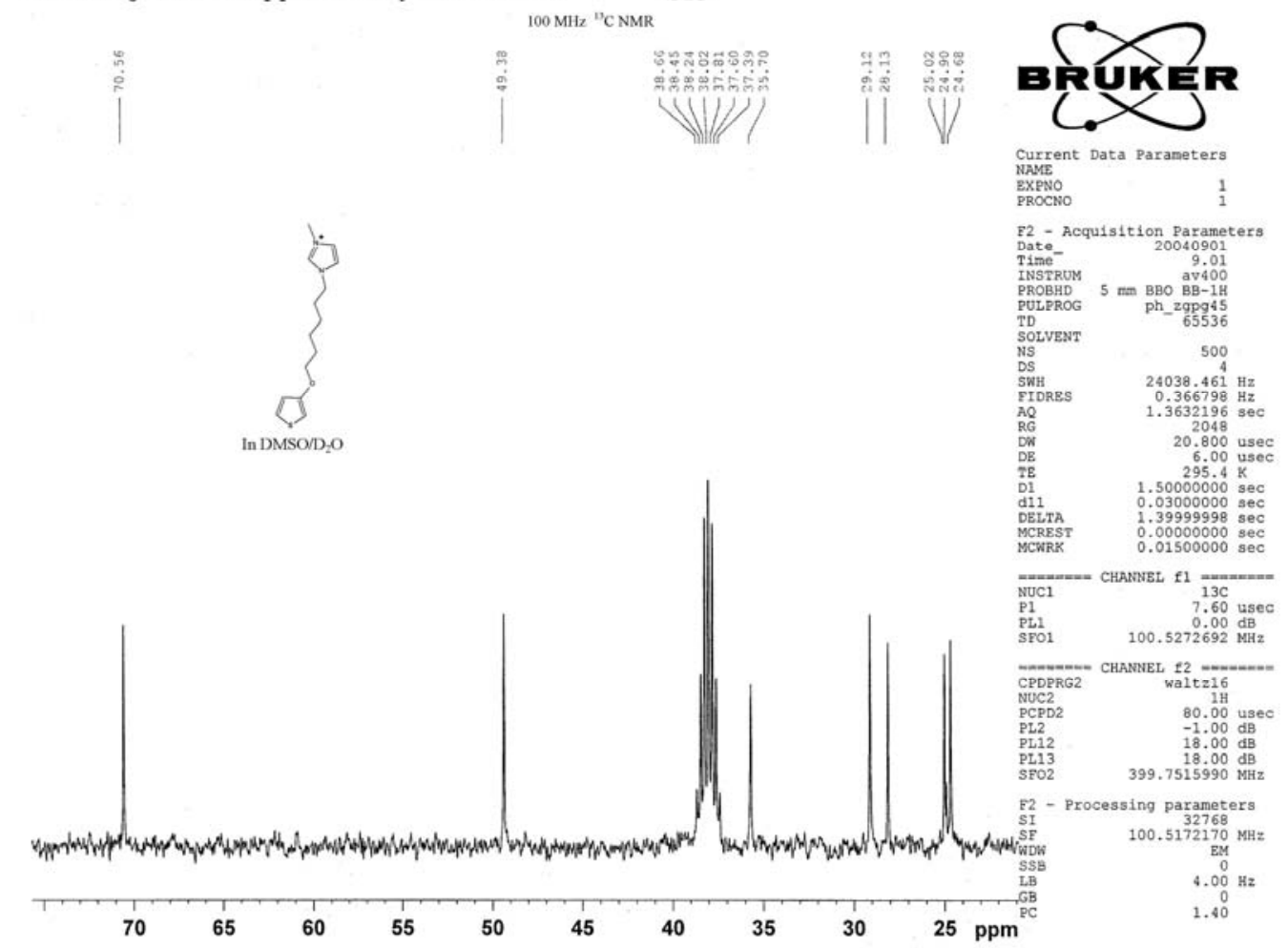




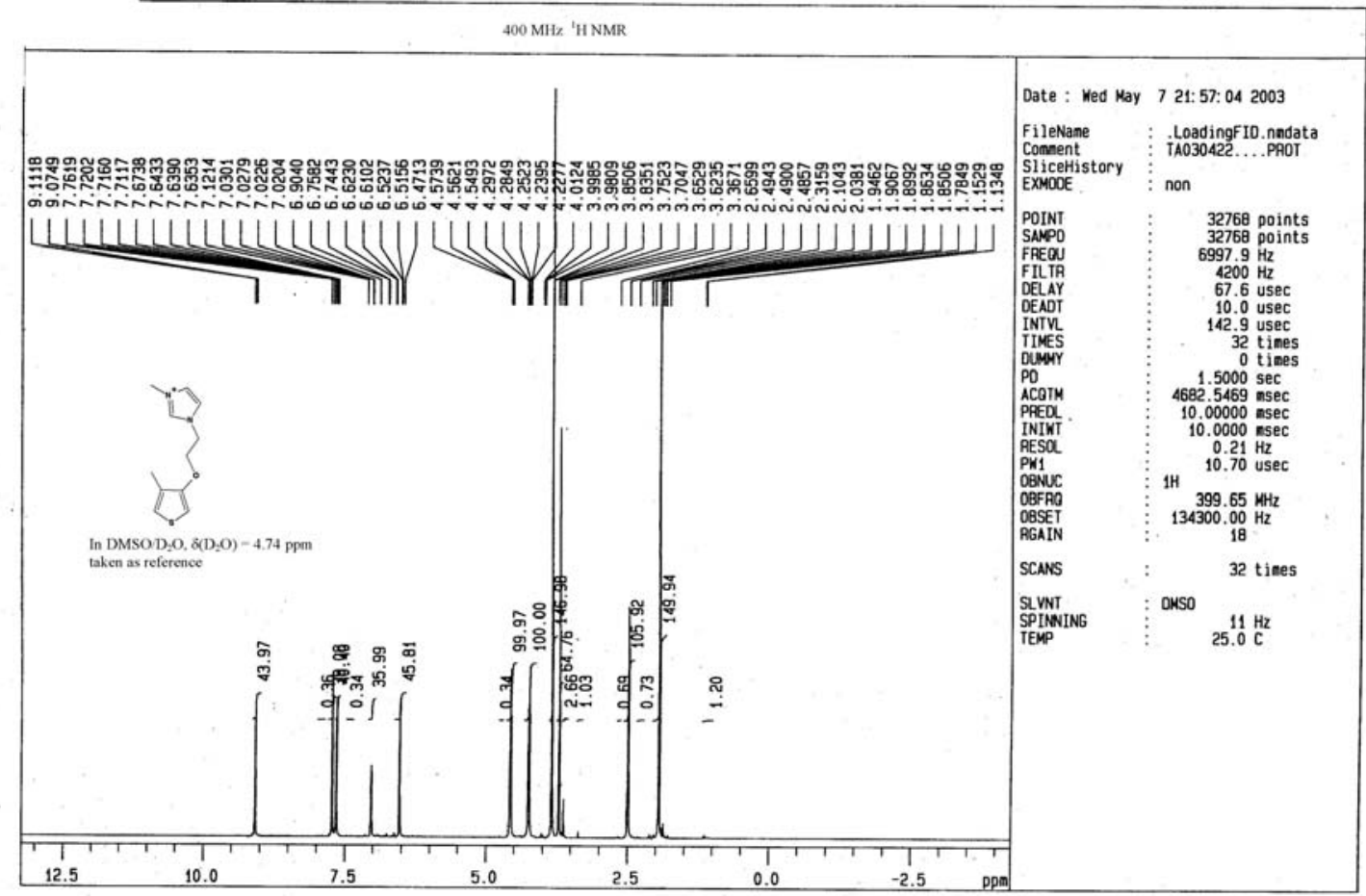

Viinikanoja et al : Supplementary Information

S17

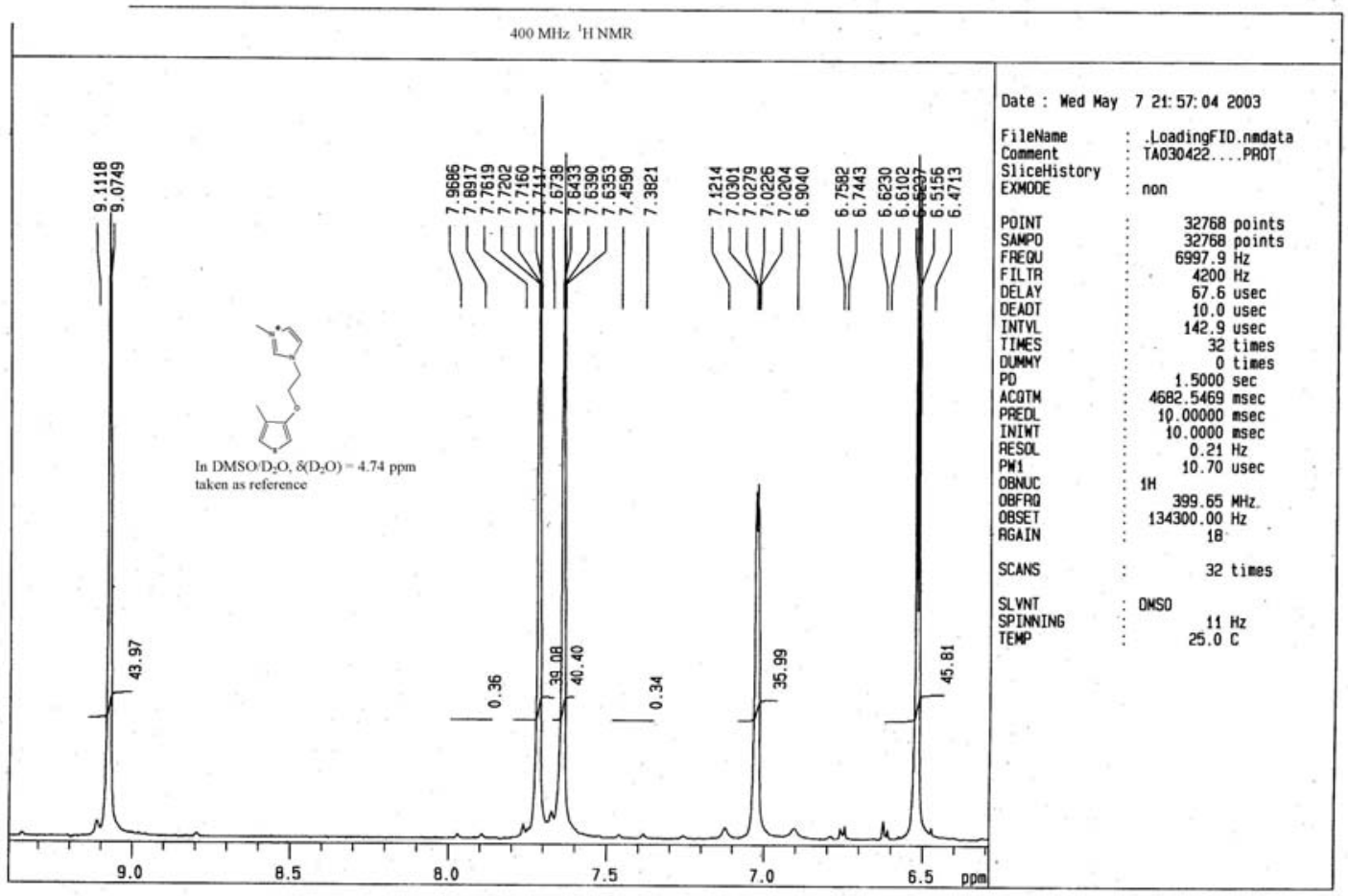




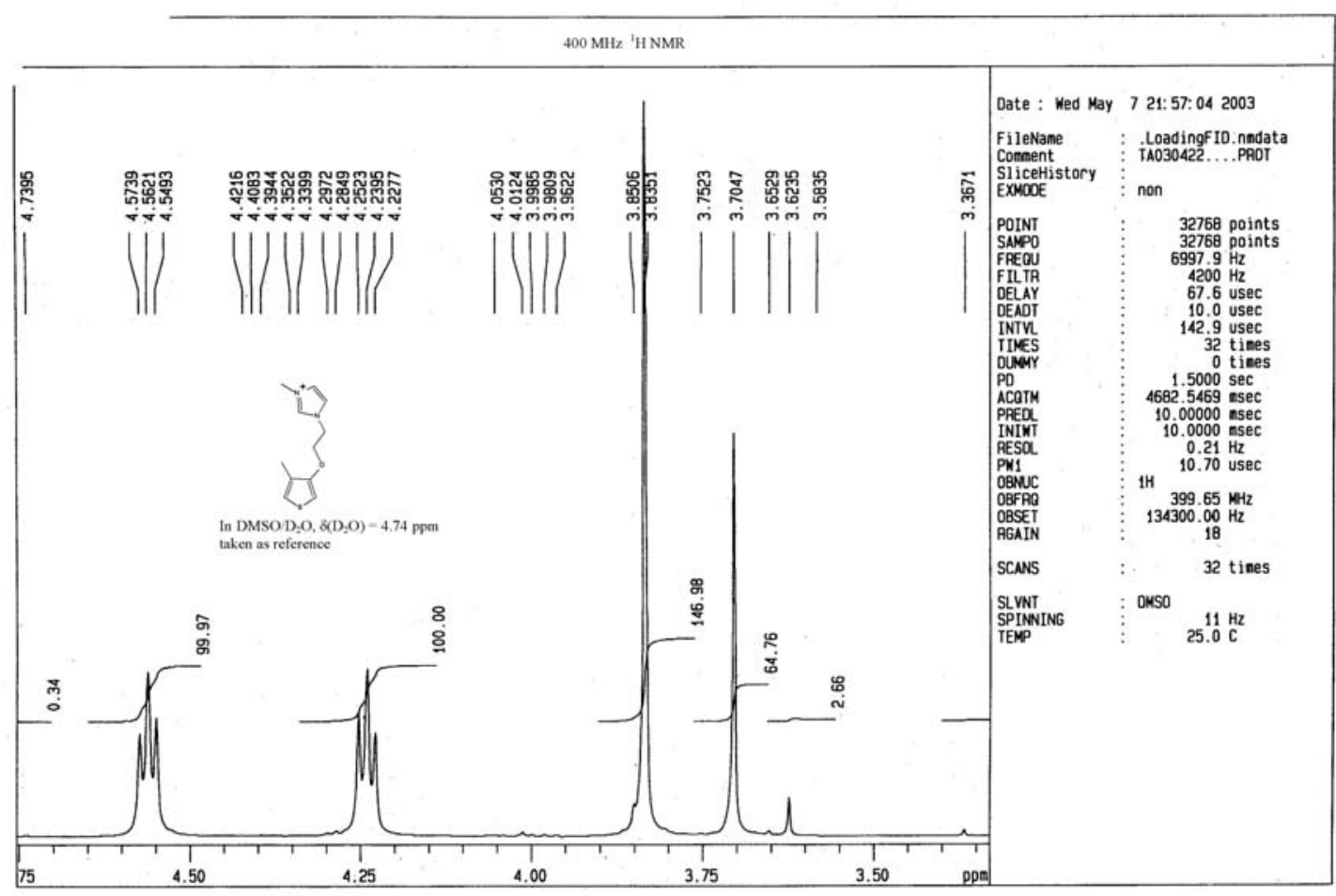

Viinikanoja et al : Supplementary Information

S19

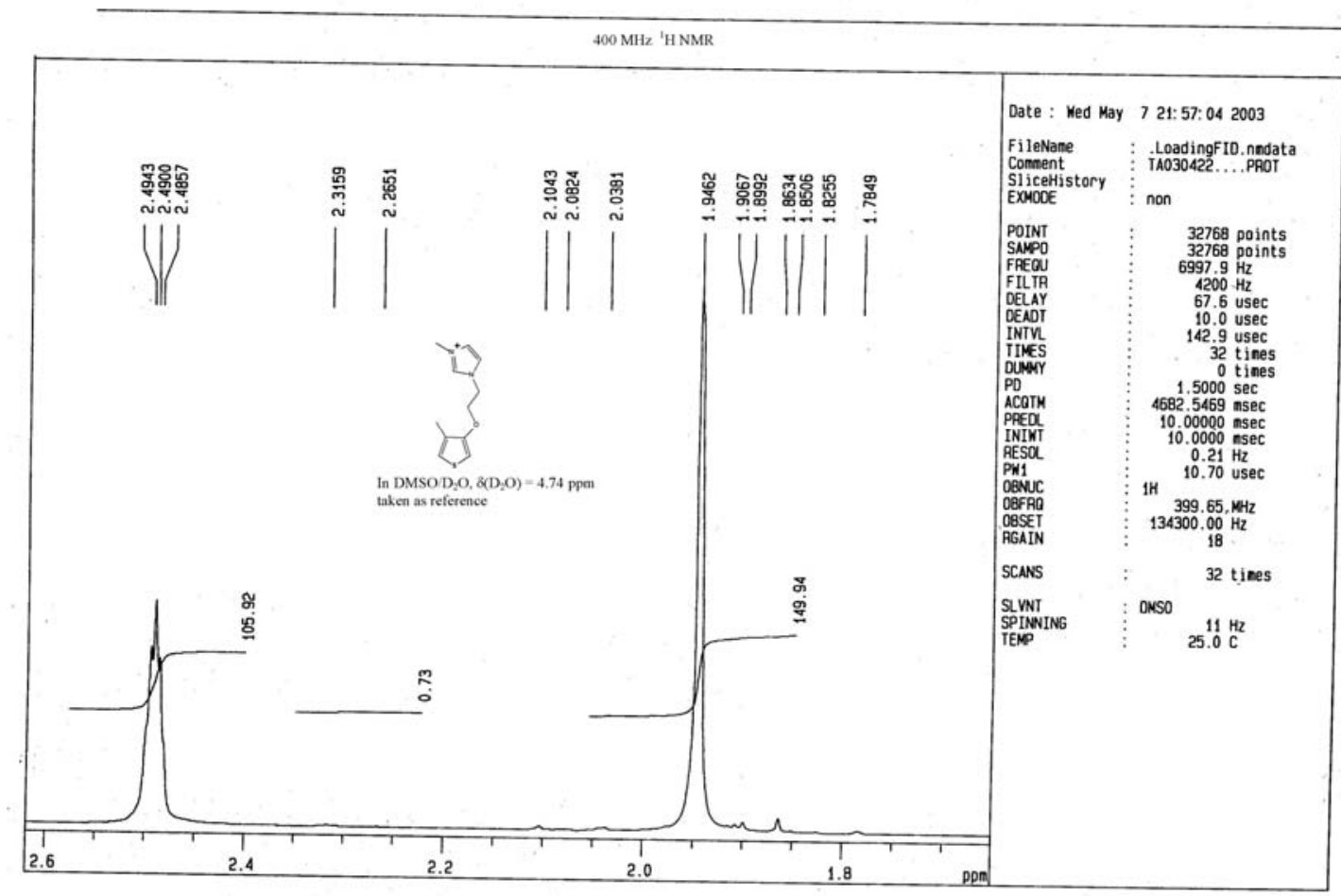



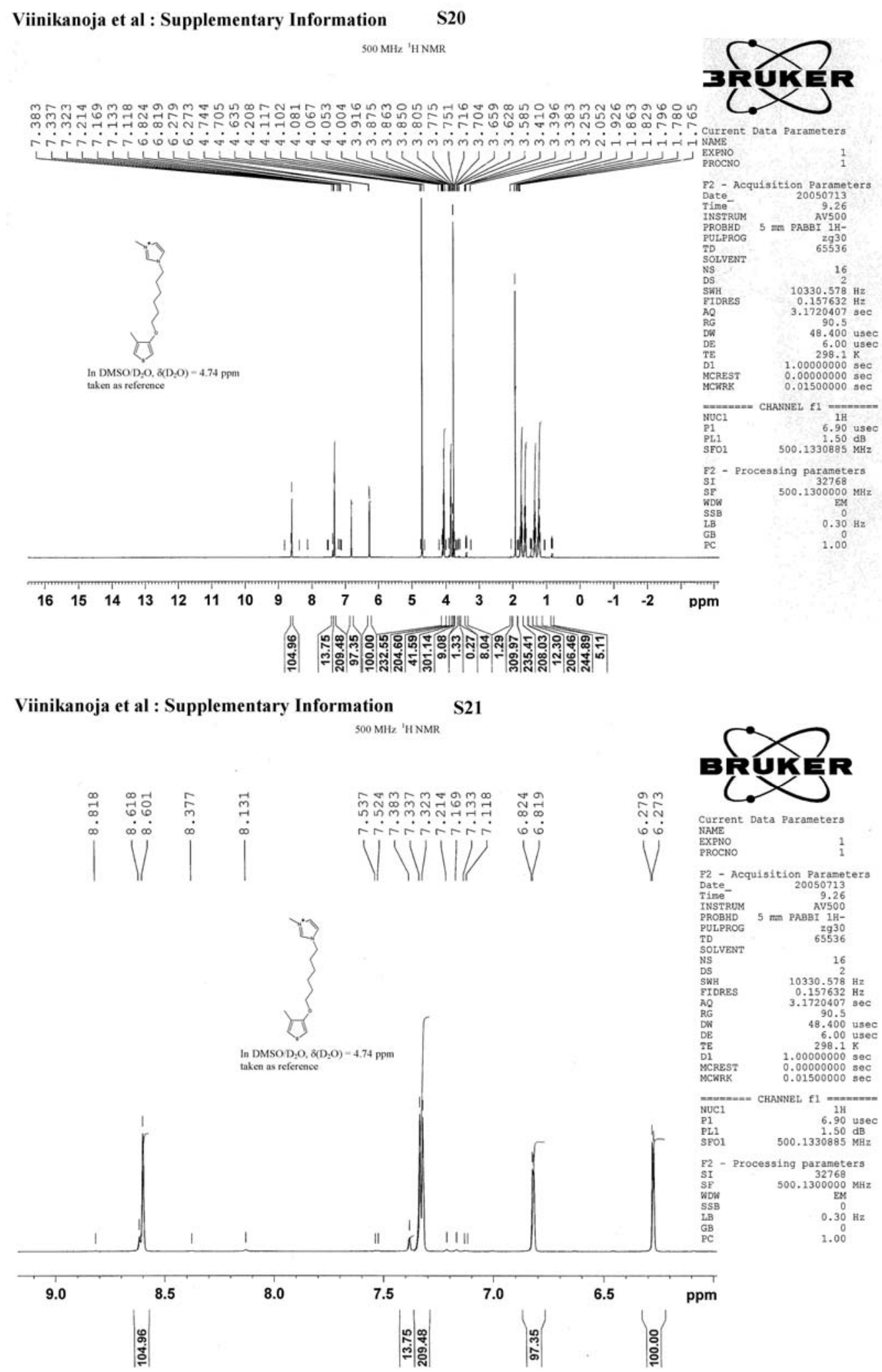


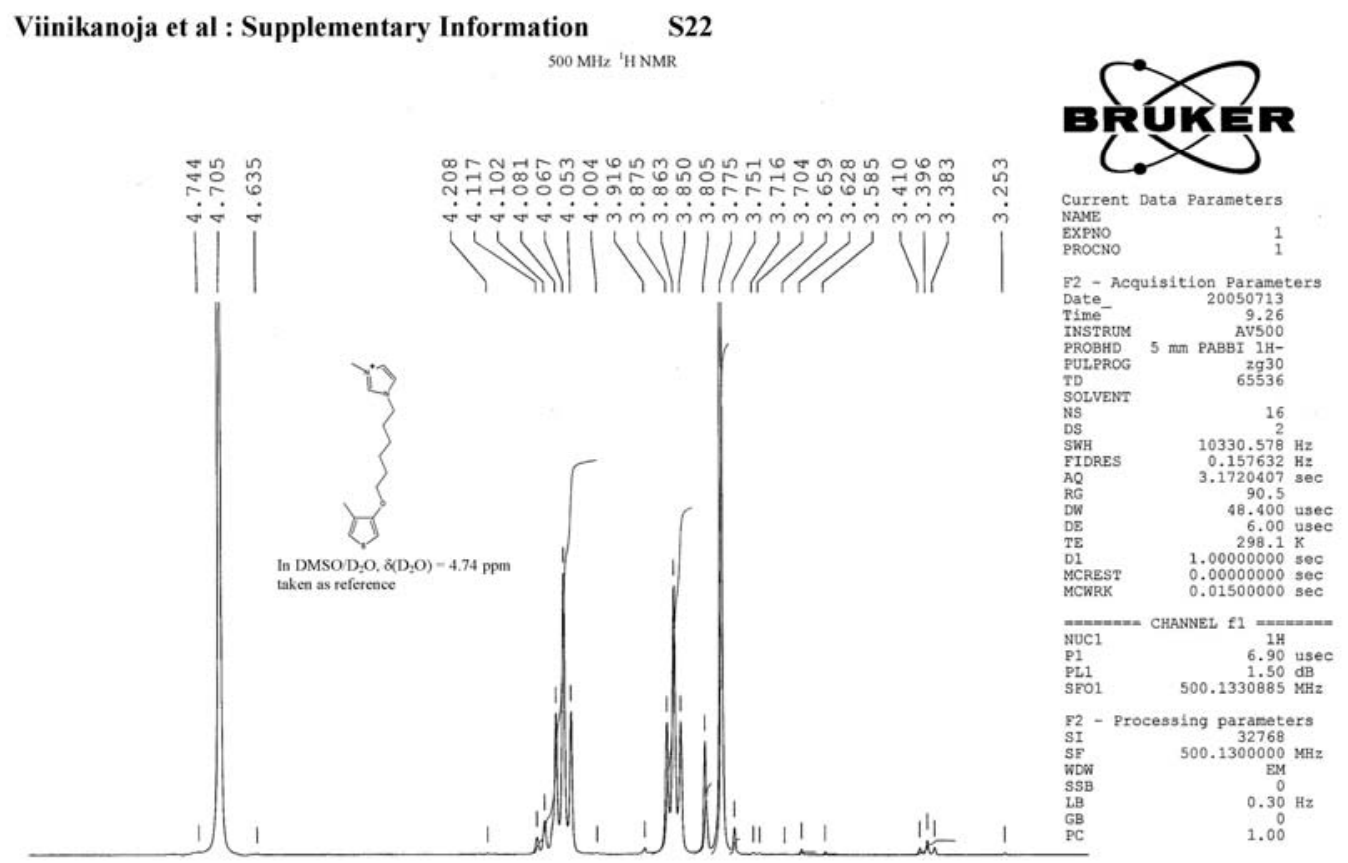

$\begin{array}{llllllllllllllllllll}5.0 & 4.9 & 4.8 & 4.7 & 4.6 & 4.5 & 4.4 & 4.3 & 4.2 & 4.1 & 4.0 & 3.9 & 3.8 & 3.7 & 3.6 & 3.5 & 3.4 & 3.3 & \mathrm{ppm}\end{array}$

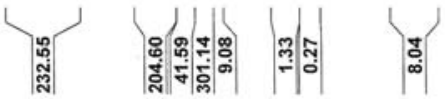

Viinikanoja et al : Supplementary Information

$\mathbf{S 2 3}$

$500 \mathrm{MHz}$ 'H NMR

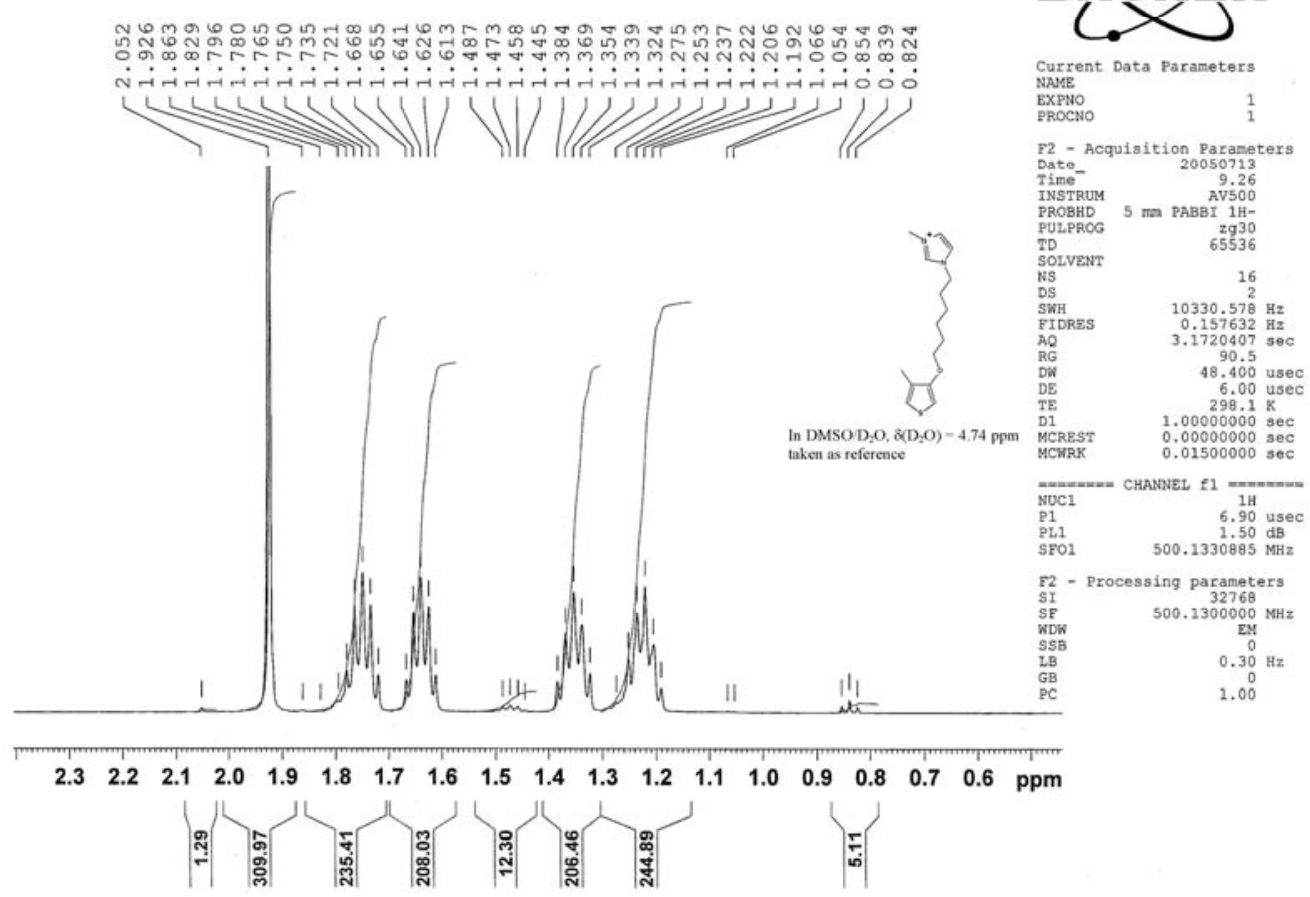


Viinikanoja et al : Supplementary Information S24

IR-spectrum (in $\mathrm{KBr}$ ) of P3TOPIM and the tentative assignment of the some major bands (peak positions in S29). The inset shows the region $3200-2800 \mathrm{~cm}^{-1}$ after the background correction.

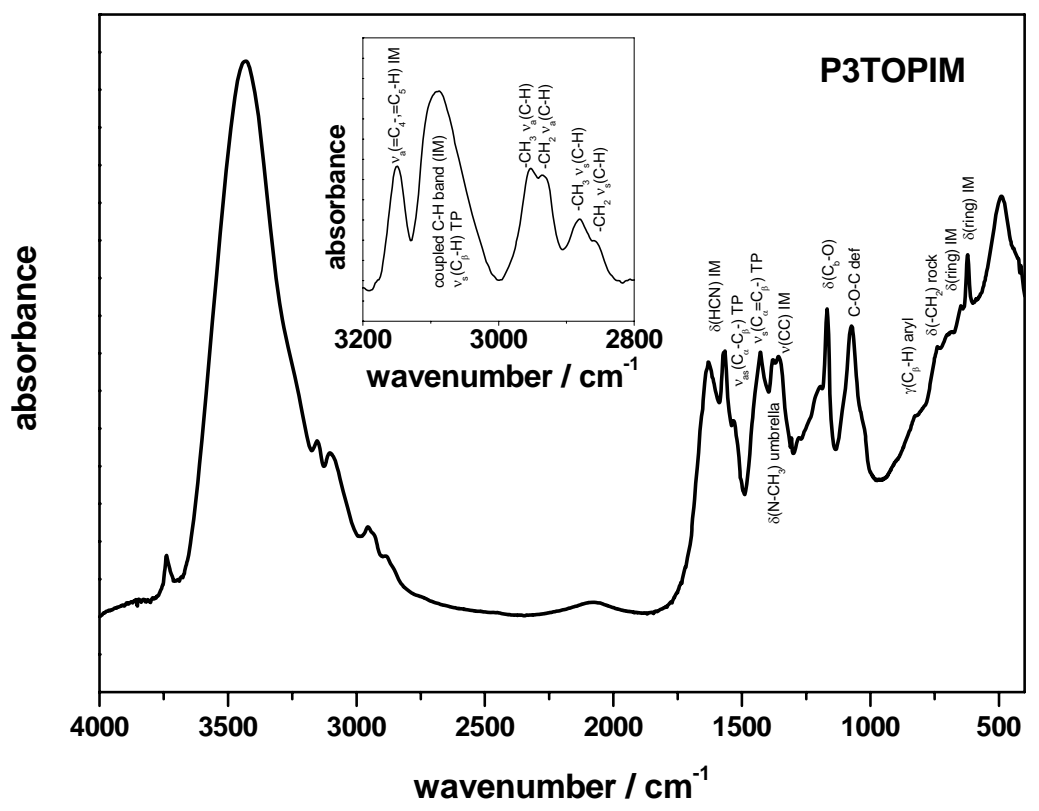

Viinikanoja et al : Supplementary Information $\quad$ S25

IR-spectrum (in $\mathrm{KBr}$ ) of P3TOHIM and the tentative assignment of the some major bands (peak positions in S29). The inset shows the region $3200-2800 \mathrm{~cm}^{-1}$ after the background correction.

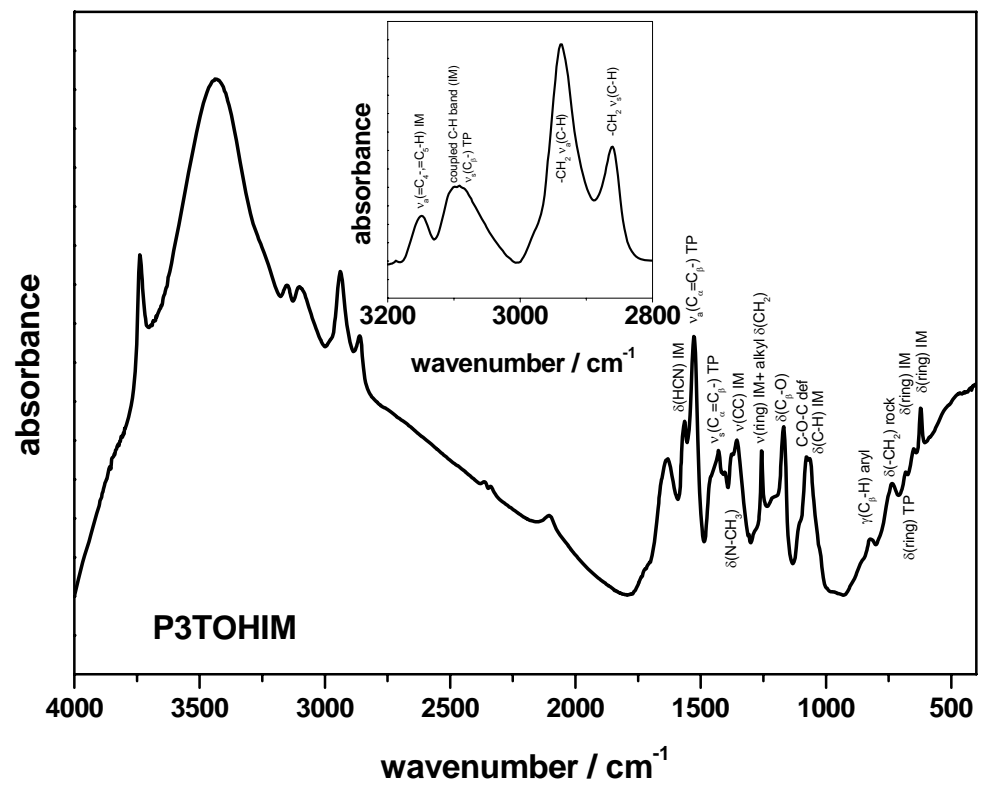


Viinikanoja et al : Supplementary Information S26

IR-spectrum (in $\mathrm{KBr}$ ) of P4Me-3TOEIM and the tentative assignment of the some major bands (peak positions in S29). The inset shows the region $3200-2800 \mathrm{~cm}^{-1}$ after the background correction.

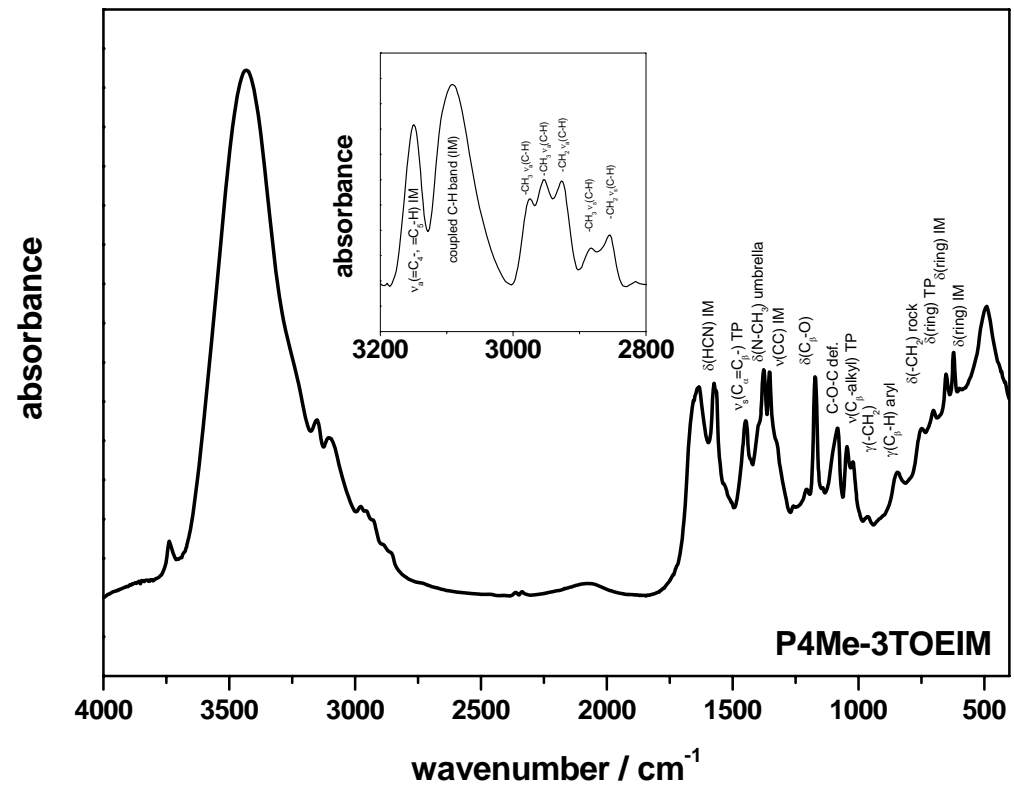

Viinikanoja et al : Supplementary Information $\quad$ S27

IR-spectrum (in $\mathrm{KBr}$ ) of P4Me-3TOHIM and the tentative assignment of the some major bands (peak positions in S29). The inset shows the region $3200-2800 \mathrm{~cm}^{-1}$ after the background correction.

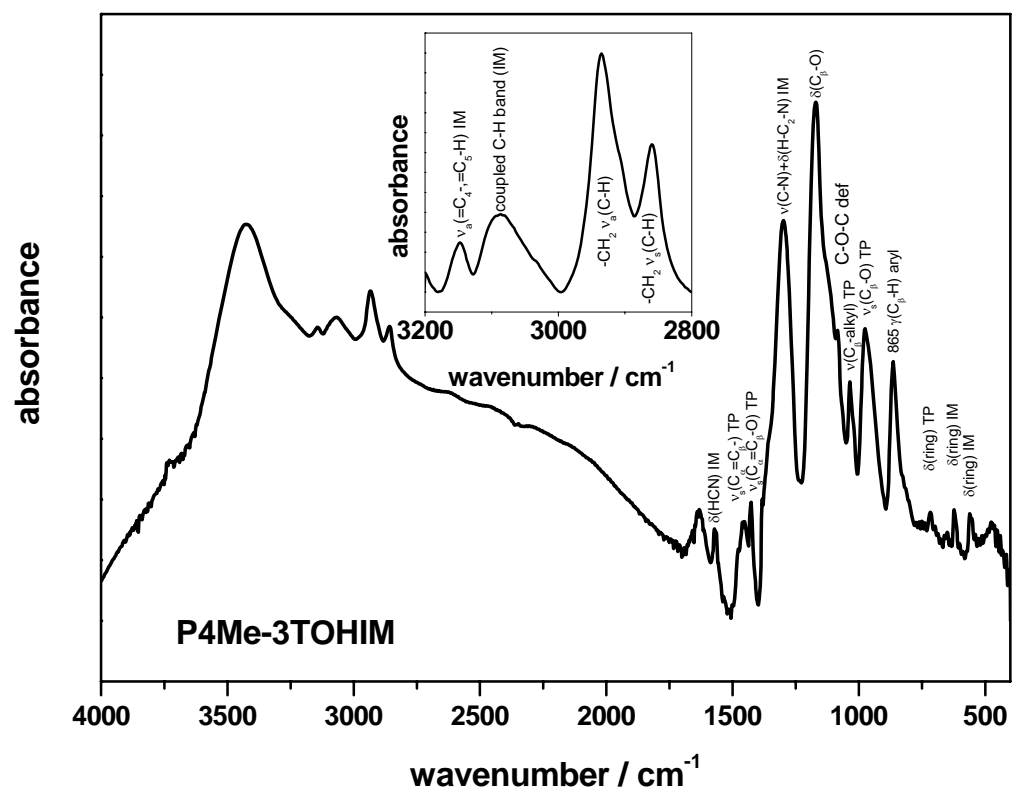


PM-IRRAS spectra of (PT/PSS) 10 alkyl C-H stretching region. In addition to methyl and methylene bands of film constituents, Fermi resonance bands (FR) and a band indicating structure with an all-trans arrangement is shown.

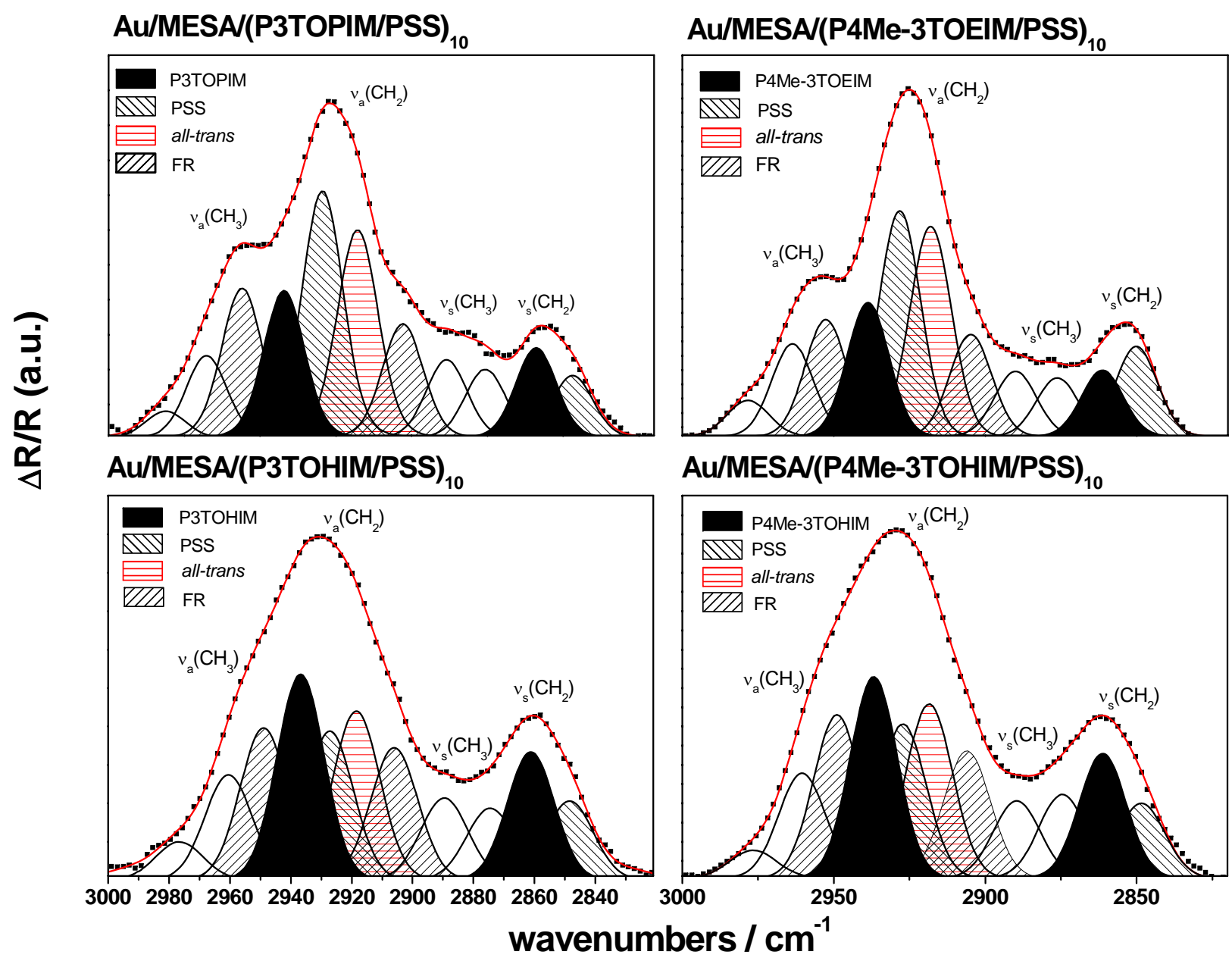


Table 1. Peak positions $\left(\mathrm{cm}^{-1}\right)$ and tentative assignments for P4Me-3TOEIM, P3TOPIM, P3TOHIM and P4Me3 TOHIM in bulk samples (in KBr) and in the (PT/PSS) 10 films (FTIR and PM-IRRAS spectra, respectively).

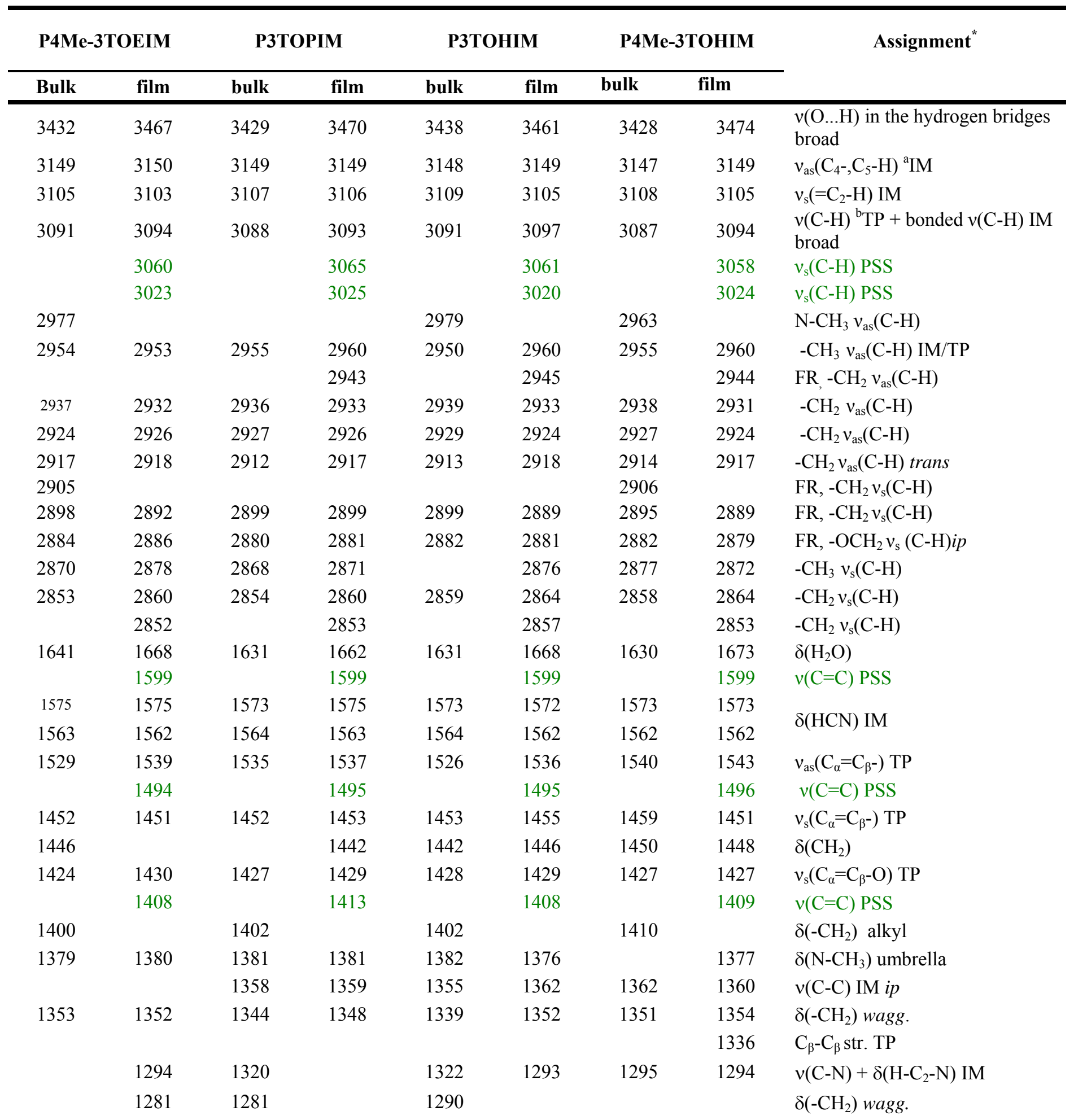




\begin{tabular}{|c|c|c|c|c|c|c|c|c|}
\hline \multicolumn{2}{|c|}{ P4Me-3TOEIM } & \multicolumn{2}{|c|}{ P3TOPIM } & \multicolumn{2}{|c|}{ Р3ТОНІМ } & \multicolumn{2}{|c|}{ Р4Ме-3ТОНІМ } & \multirow[t]{2}{*}{ Assignment } \\
\hline bulk & film & bulk & film & bulk & film & bulk & film & \\
\hline 1260 & $\begin{array}{l}1265 \\
1222 \\
1199\end{array}$ & & $\begin{array}{l}1222 \\
1201\end{array}$ & 1257 & $\begin{array}{l}1264 \\
1223 \\
1202\end{array}$ & 1260 & $\begin{array}{l}1221 \\
1202\end{array}$ & $\begin{array}{l}\text { IM } v(\text { ring })+\text { alkyl } \delta\left(\mathrm{CH}_{2}\right) \\
v_{\text {as }}\left(\mathrm{SO}_{3}^{-}\right) \text {PSS }\end{array}$ \\
\hline 1096 & & 1102 & & 1110 & 1109 & 1104 & 1107 & $\mathrm{IM} \delta\left(\mathrm{N}-\mathrm{CH}_{3}\right)+\delta(\mathrm{CNC})$ \\
\hline 1080 & $\begin{array}{l}1085 \\
1056\end{array}$ & 1074 & $\begin{array}{l}1077 \\
1051\end{array}$ & 1081 & $\begin{array}{l}1086 \\
1058\end{array}$ & 1082 & $\begin{array}{l}1087 \\
1046\end{array}$ & $\begin{array}{l}\mathrm{C}-\mathrm{O}-\mathrm{C} \text { def. TP } \\
\mathbf{v}_{\mathrm{s}}\left(\mathrm{SO}_{3}^{-}\right) \mathrm{PSS}\end{array}$ \\
\hline 1047 & 1036 & & 1036 & 1037 & 1036 & 1036 & 1036 & $\begin{array}{l}v\left(\mathrm{C}_{\beta} \text {-alkyl }\right) \mathrm{TP} \\
v_{\mathrm{s}}\left(\mathrm{SO}_{3}^{-}\right) \text {PSS }\end{array}$ \\
\hline 752 & & 740 & & 740 & & 820 & & $\delta\left(-\mathrm{CH}_{2}\right)$ rock \\
\hline 704 & & 706 & & 683 & & 718 & & $\delta$ (ring) TP \\
\hline 652 & & 648 & & 650 & & 653 & & $\delta$ (ring) IM \\
\hline 622 & & 622 & & 623 & & 621 & & $\delta($ ring $) \quad$ IM \\
\hline
\end{tabular}

${ }^{\mathrm{a}} \mathrm{IM}=$ methyl imidazolium,${ }^{\mathrm{b}} \mathrm{TP}=$ thiophene, PSS modes

*References: $\mathrm{TP}^{1}, \mathrm{PSS}^{2}, \mathrm{IM}^{3}$

(1) (a) Pron, A.; Louarn, G.; Lapkowski, M.; Zagórska. M.; Glowczyk-Zubek, J.; Lefrant, S. Macromolecules 1995, 28, 4644 (b) Villa, E.; Agosti, E.; Castiglioni, C.; Gallazzi, M. C.; Zerbi, G. J. Chem. Phys. 1996, 105, 9461 (c) Louarn, G.; Buisson, J. P.; Lefrant, S.; Fichou, D. J. Phys. Chem. 1995, 99, 11399 (d) Garreau, S.; Louarn, G.; Buisson, J. P.; Froyer, G.; Lefrant, S. Macromolecules 1999, 32, 6807 (e) Louarn, G.; Mevellec, J. Y.; Buisson, J. P.; Lefrant, S. Synth. Met. 1993, 55-57, 587 (f) Zerbi, G.; Chierichetti, B.; Ingänas, O. J. Chem. Phys. 1991, 94, 4646 (g) Casado, J.; Katz, H. E.; Hernández, V.; López Navarrete, J. T. Vib. Spectrosc. 2002, 30, 175 (h) Liedberg, B.; Yang, Z.; Engquist, I.; Wirde, M.; Gelius, U.; Götz, G.; Bäuerle, P.; Rummel, R. -M.; Ziegler, Ch; Göpel, W. J. Phys. Chem. B 1997, 101, 5951 (i) Garreau, S.; Leclerc, M.; Errien, N.; Louarn, G. Macromolecules 2003, 36, 692 (j) Buga, K.; Kepczynska, K.; Kulszewicz-Bajer, I.; Zagórska, M.; Demadrille, R.; Pron, A.; Quillard, S.; Lefrant, S. Macromolecules 2004, 37, 769 (k) Garreau, S.; Louarn, G.; Buisson, J. P.; Froyer, G.; Lefrant, S. Macromolecules 1999, 32, 6807 (l) Louarn, G.; Trznadel, M.; Buisson, J. P.; Laska, J.; Pron, A.; Lapkowski, M.; Lefrant, S. J. Phys. Chem. 1996, 100, 12532

(2) Zundel, G. in Hydration and Intermolecular Interaction; Academic Press, New York, 1969

(3) (a) Cordes, M.; Walter, J. L. Spectrochim. Acta 1968, 24A, 237 (b) Sadlej, J.; Jaworski, A.; Miaskiewicz, K. J. Mol. Struct. 1992, 274, 247 (c) Bukowska, J.; Kudelski, A.; Jackowska, K. J. Electroanal. Chem. 1991, 309, 251 (d) Bellocq, A.-M.; Garrigou-Lagrange, C. Spectrochim. Acta 1971, 27A, 1091 (e) Dieter, K. M.; Dymek, C. J, Jr.; Heimer, N. E.; Rovang, J. W.; Wilkes, J. S. J. Am. Chem. Soc. 1988, 110, 2722 (f) Tait, S.; Osteryoung, R. A. Inorg. Chem. 1984, 23, 4352 (g) Elaiwi, A.; Hitchcock, P. B.; Seddon, K. R.;Srinivasan, N.; Tan, Y. M.; Welton, T.; Zora, J. A. J. Chem. Soc., Dalton Trans. 1995, 3467 (h) Kölle, P.; Dronskowski, R. Inorg. Chem. 2004, 43, 2803 
Viinikanoja et al : Supplementary Information

30

Density of PT-based aggregates in (PT/PSS) 10 multilayers $(125-200 \mathrm{~nm}$ range).

\begin{tabular}{|l|l|}
\hline \multicolumn{1}{|c|}{ Film } & Density $\left(\right.$ particles $\left./ \mu \mathrm{m}^{2}\right)$ \\
\hline$(\text { P3TOPIM/PSS })_{10}$ & 32 \\
\hline$(\text { P4Me-3TOEIM/PSS })_{10}$ & 26 \\
\hline$(\text { P3TOHIM/PSS })_{10}$ & 35 \\
\hline$(\text { P4Me-3TOHIM/PSS })_{10}$ & 125 \\
\hline
\end{tabular}

